\title{
Self-organized criticality of molecular biology and thermodynamic analysis of life system based on optimized particle swarm algorithm
}

\author{
Jin $\mathrm{Li}^{1 *}$, Fang $\mathrm{Xie}^{2}$ \\ ${ }^{1}$ Collge of Arts and Sciences, Shanghai Maritime University, Shanghai, 200136, China \\ ${ }^{2}$ Sports Teaching and Research Section, Senior High School of Hongkou, Shanghai, 200438, China
}

*Correspondence to: smuyari7979@163.com

Received February 12, 2020; Accepted May 6, 2020; Published May 15, 2020

Doi: http://dx.doi.org/10.14715/cmb/2020.66.2.29

Copyright: $\odot 2020$ by the C.M.B. Association. All rights reserved.

\begin{abstract}
In order to improve the thermodynamic analysis and prediction ability of biological self-organized criticality and life system, a prediction model of biological self-organized criticality and thermodynamic characteristics of life system based on particle swarm optimization neural network is proposed. Fuzzy regression parameter fusion model is adopted to rearrange the statistical prior data of biological self-organized criticality and thermodynamic characteristics of life system, neural network training method is adopted to extract principal component characteristics of rearranged biological self-organized criticality and thermodynamic information flow of life system, and optimized particle swarm algorithm is adopted to carry out feature selection and self-organized supervised learning on extracted principal component characteristics, thus realizing accurate prediction of biological self-organized criticality and thermodynamic characteristics of life system. The simulation results show that the prediction accuracy of biological self-organization criticality and thermodynamic characteristics of life system using this model is high, the prior sample knowledge required is relatively small, and the reliability of biological self-organization criticality characteristics analysis is guaranteed.
\end{abstract}

Keywords: Biological self-organization criticality; Life system; Thermodynamics; Forecast.

\section{Introduction}

One of the differences between living and nonliving organisms is the existence of instructions and controls for the self-replication of biological structures, the growth and self-adaptability showed by the life activity are a series of macroscopical performances produced by the environment and repeated iteration through the genetic rule-making program. It makes life activities only under certain boundary conditions, many really complex biological phenomena are actually in the critical state to show up (1).

Biological self-organization refers to a kind of self-organization and self-construction of organisms themselves, which are closely connected with the induction of the natural environment. It is not isolated self-organization that we often say is not affected by the environment. Biological 
self-organization reflects the role of organisms themselves in their evolution. Biological self-organization refers to self-organization closely connected with the induction of natural environment, rather than isolated self-organization unrelated to the environment. Biological self-organization focuses on the organization and is precise (1), orderly and regular due to organization. When the natural environment changes, organisms will reorganize themselves to form new traits. Biological self-organization is not random or irregular but follows strict natural laws. Various self-organized changes of living macromolecules strictly follow complex physical and chemical laws and are related to many factors in the surrounding microenvironment, such as molecular polarity, solubility (2), acidity and basicity, attraction, repulsion, charge amount, thermal stability, inorganic salts, temperature, etc. The variation of living macromolecules is just like the change of substance molecules in chemical reactions. It is orderly and takes place under certain environmental conditions, but the variation of living macromolecules is only more complicated. The evolution of chromosome structure and morphology is not random, but organized and formed in a specific microenvironment, following certain rules. Nuclear matrix, nuclear skeleton and small molecular substances in microenvironment will all affect the tissue construction of chromosomes (3).

In every advanced organism, there are thousands of cells. Each cell has a complex and fine microstructure. Each structure consists of tens of millions of molecules. Different cells, tissues and organs perform different physiological functions (4). Molecular biology research shows that all kinds of biological life activities are a huge and complex project, and their precise and orderly operation depends on the strict, precise and orderly self-organization of organisms. Biology is like a precision instrument. In order for the instrument to work normally, all parts must exist at the same time (5). If any part is randomly changed, the instrument cannot work normally. Studies have shown that even the composition of a particularly simple bacterial flagellum is so complex that the probability of simultaneously producing all the components of the flagellum through random mutation is infinitesimal. For more complex biological structures, such as the eyes of animals, it is impossible to generate them through random variation, and biological self-organization provides the possibility for the formation of these structures.

Biological self-organization is a complex process, involving the changes of many kinds of material molecules. When the natural environment changes, organisms will accept the information of the change of the natural environment at different levels of structural level and material molecular level to self-organization and improve, forming new variation characters, which are manifested in the high adaptation of new characters to the environment, and the coordination and unity of organisms and the natural environment.

The function of biological self-organization is very extensive. Every cell, tissue, organ, system and so on can feel the change information of the natural environment so as to make corresponding processing for the information. The corresponding structure and material molecules change their self-organization again, which shows the corresponding biological variation. All kinds of character variation, structural change and molecular change are the result of self-organization. In a broad sense, all kinds of reactions in an organism can be regarded as a kind of biological self-organization, which is the general term of all reactions in an organism.

The change of a certain level of material molecules in the organism will induce the next level of material molecules to reorganize and produce new variation molecules, and the new variation molecules will re inducing the next level of material molecules to reorganize and change, forming 
the chain reaction of molecules. The complex changes of induction, self-organization, induction and self-organization are carried out among different levels of molecules. The changes in these different levels of material molecules are complex, interrelated and collaborative. The change of the internal environment will induce the change of cytoplasm, the change of cytoplasm will induce the change of the nuclear matrix, and the change of the nuclear matrix will induce the change of the chromosome, which involves complex signal conduction and molecular reaction.

The data of biological self-organized criticality and thermodynamic characteristics of the life system are essentially a set of linearly related information parameters (6). It is feasible to predict biological self-organized criticality and thermodynamic characteristics of the life system by using information processing technology. The traditional methods mainly adopt prediction methods such as decision tree prediction method, neural network prediction algorithm and unsupervised learning training method (7). The parallel processing algorithm is used to predict the biological self-organization criticality and thermodynamic characteristics of life systems with a massive information scale. By processing and analyzing the sample information flow, combining correlation test and feature extraction, accurate prediction is realized and prediction efficiency is improved. However, the traditional algorithm has many problems, such as more prior sample knowledge and higher calculation cost, etc. In this regard, in this paper, a prediction model of biological self-organized criticality and thermodynamic characteristics of the life system based on particle swarm optimization neural network is proposed. It is effective to improve the prediction algorithm of biological self-organized criticality and thermodynamic characteristics of the life system and to optimize the design of the prediction model.

\section{Basic Definitions}

\section{Exponential Spectrum Calculation of Statistical Biological Self-organized Criticality and Thermodynamic Characteristic Data of Life System}

Self-organization is the process and performance of an open system producing a new macroscopically ordered structure with the cooperation of a large number of subsystems. At present, many typical examples have been studied, such as Besnard convection, dissipative structure and coherent structure, in 1987, Santa Fe Institute member Buck and his colleagues put forward the complex adaptive system theory and the Self-organized criticality, which provide a good answer to the behavior of such complex systems and mechanisms, the previous dissipative structure theory and synergetics focused on the formation and emergence of the dissipative structure system, the existing conditions, the environment and General Dynamics. The complexity of the life system is manifested in the following five aspects:

1. Life is complex and cannot be made up of one component;

2. There is a wide association between the components;

3. The life system has the order and the stratification;

4. The whole is greater than the sum of its parts, the so-called "Emergence";

5. The system is open.

In order to accurately predict the biological self-organized criticality and thermodynamic characteristics of the life system, an information processing algorithm is adopted to analyze sample data and rearrange information characteristics to construct a prediction model. Assuming that the prior knowledge of statistical biological self-organized criticality and thermodynamic 
characteristic data time series sampling of the life system is expressed as $\left\{x_{i}\right\}_{i=1}^{N}$ the original biological self-organized criticality and thermodynamic characteristic data of the life system have Gaussian process characteristics linearly related. According to the rank of the sequences, data arrangement analysis is carried out to obtain the original sequence amplitude of the biological self-organization criticality and the thermodynamic characteristics of the life system (8), discriminant statistics of statistical data are constructed to generate individual dimension vectors, and a fuzzy regression parameter fusion model is adopted to divide the characteristic initial solution of the original sequence of the biological self-organization criticality and the thermodynamic characteristics of the life system into $\mathrm{N}$ parts $\mathbf{x}^{(1)}, \mathbf{x}^{(2)}, \ldots, \mathbf{x}^{(N)}$, namely $\mathbf{x}^{(0)}=\bigcup_{i=1}^{N} \mathbf{x}^{(i)}$, According to the rank of the biological self-organized criticality and the thermodynamic characteristic sequence of the life system, the original biological self-organized criticality and thermodynamic characteristic data of the life system are arranged, and a fitting model for statistical biological self-organized criticality and thermodynamic characteristic data of the life system is obtained and expressed as follows:

$$
x_{n}=\varphi_{0}+\sum_{i=1}^{p} \varphi_{i} x_{n-i}+\sum_{j=0}^{q} \theta_{j} \eta_{n-j}
$$

Where $\left\{\eta_{i}\right\}$ is the linear correlation Gaussian random variable with a mean value of 0 and a variance, $\varphi_{0}, \varphi_{1}, \varphi_{2}, \cdots \varphi_{p}$, is called the time inversion irreversible characteristic decomposition coefficient of biological self-organization criticality and thermodynamic characteristics of the life system, $\theta_{1}, \theta_{2}, \cdots \theta_{q}$ is called the average coefficient of sliding time window, and the exponential spectrum calculation iteration formula for statistical biological self-organization criticality and thermodynamic characteristic data of the life system is obtained as follows:

$$
\begin{aligned}
& \max _{x_{a, b, d, p}} \sum_{a \in \mathrm{A}} \sum_{b \in \mathrm{B}} \sum_{d \in \mathrm{D}} \sum_{p \in \mathrm{P}} x_{a, b, d, p} V_{p} \\
& \text { s.t. } \quad \sum_{a \in \mathrm{A}} \sum_{d \in \mathrm{D}} \sum_{p \in \mathrm{P}} x_{a, b, d, p} R_{p}^{b w} \leq K_{b}^{b w}(\mathrm{~S}), b \in \mathrm{B}
\end{aligned}
$$

In the two-dimensional main feature space that counts the self-organized criticality of organisms and the distribution of thermodynamic feature data of life systems, the fuzzy neural network is used for feature training through exponential spectrum calculation, and particle swarm optimization is combined for learning optimization to improve prediction accuracy (9).

\section{Information rearrangement calculation}

The fuzzy regression parameter fusion model is used to rearrange the statistical prior data of biological self-organization criticality and thermodynamic characteristics of the life system. Assuming that the population of the particle swarm in the fuzzy regression parameter fusion model is, wherein the length of the biological self-organization criticality corresponding to the position of the first particle swarm and the thermodynamic characteristics time series of the life system is (10), the luciferin value is calculated by combining the phase randomized Fourier transform (RPFT) method, and thus the particle swarm obtains the information rearrangement 
Jin Li and Fang Xie

update formula with the same visual field as the original data as:

$$
\begin{aligned}
R_{d}^{i}(t+1) & =\min \left\{R_{s}, \max \left\{0, R_{d}^{i}(t)+\beta\left(n_{t}-\left|N_{i}(t)\right|\right)\right\}\right\} \\
N_{i}(t) & =\left\{j:\left\|x_{j}(t)-x_{i}(t)\right\|<R_{d}^{i} ; l_{i}(t)<l_{j}(t)\right\}
\end{aligned}
$$

Wherein $x_{j}(t)$ represents the position of the prior data of biological self-organization criticality and thermodynamic characteristics of the life system in the J-th particle swarm of the 1st generation, represents the average value of the optimal positions of the I-th particle swarm of the J-th generation, the speed and position of adjacent particle swarms updating themselves, and the global optimal position distribution of the biological self-organization criticality and thermodynamic particle swarm of the life system within the scope $R_{d}^{i}$ of the thermodynamic prediction of the biological self-organization criticality and the life system is as follows:

$$
p_{i j}(t)=\frac{l_{j}(t)-l_{i}(t)}{\sum_{k \in N_{i}(t)} l_{k}(t)-l_{i}(t)}
$$

When the current biological self-organization criticality meets the convergence condition (11-13), the particle swarm location update formula:

$$
x_{i}(t)=x_{i}(t-1)+s\left(\frac{x_{j}(t-1)-x_{i}(t-1)}{\left\|x_{j}(t-1)-x_{i}(t-1)\right\|}\right)
$$

Particle swarm performs distribution feature search of biological self-organization criticality and thermodynamic characteristics of a life system in a D-dimensional space of a fuzzy regression parameter fusion model, and adopts fluorescein values as search conditions, wherein the search formula is as follows:

$$
l_{i}(t+1)=(1-\rho) l_{i}(t)+\gamma J\left(x_{i}(t+1)\right)
$$

By fluorescein search, the prior data information rearrangement of biological self-organization criticality and thermodynamic characteristics of the life system is obtained. The specific implementation is described as follows:

1) Initialization. Assume that the maximum number of iterations for the biological self-organization criticality and thermodynamic prediction of the life system at a time $k-1$ is $\left\{x_{k-1}^{i}, w_{k-1}^{i}\right\}$. The adaptive function $p\left(x_{0}\right)$ is calculated to generate the initial inertia weight $\left\{x_{0}^{i}, i=1,2, \ldots \ldots \mathrm{N}\right\}$ of the particle swarm individuals, and all the particle swarm weights are $1 / \mathrm{n}$.

2)Returning to the construction of moving average model and feature sampling: if the self-organized criticality of organisms and the thermodynamic random inertia weight of life systems are small, calculate the statistical probability density function $q\left(x_{k}^{i} / x_{k-1}^{i}\right)$ and update the particle cluster.

3) Calculating fitness distribution weights of each particle swarm individual;

$$
\overrightarrow{w_{k}}=\vec{w}_{k-1}^{i} \frac{p\left(z_{k} / \bar{x}_{k}^{i}\right) p\left(\bar{x}_{k}^{i} / x_{k-1}^{i}\right)}{q\left(\bar{x}_{k}^{i} / x_{k-1}^{i}\right)}
$$

4) Calculating normalized weight value:

$$
\underset{w_{k}}{\mathfrak{q}_{k}}=\square_{k}^{N} / \sum_{i=1}^{N} w_{k}^{i}
$$


5) The current position xi replaces pi and performs global extreme value sampling: defining effective sampling, setting a threshold value $N_{\text {eff }} \approx 1 / \sum_{i=1}^{N}\left(\vec{w}_{k}\right)^{2}$, when the fitness function of the current position xi meets $N_{\text {eff }}<N_{t h}$, resampling from the $N_{\text {eff }}<N_{t h}$ set according to the global optimal solution weight to obtain a characteristic distribution set $\vec{w}_{k}$ of biological self-organization criticality and thermodynamic characteristic prior data of a life system, and redistributing the weight values (14).

6) If those run iteration times reach a preset maximum value, the obtain estimation formula for rearrangement of prior data information of biological self-organization criticality and thermodynamic characteristics of a life system is as follows:

$$
\hat{x}_{k}=\sum_{i=1}^{N} w_{k}^{i} x_{k}^{i}
$$

The prior information on thermodynamic characteristics is analyzed to generate new inertia weight values and improve the convergence of prediction.

\section{Self-organized Criticality of Biology and Optimization of Thermodynamic Characteristic Prediction Model for Life System}

On the basis of rearrangement of prior data information of biological self-organized criticality and thermodynamic characteristics of life system, optimization design of prediction model of biological self-organized criticality and thermodynamic characteristics of life system is carried out. This paper proposes a prediction model of biological self-organized criticality and thermodynamic characteristics of the life system based on particle swarm optimization neural network (15).

Particle Swarm Optimization, also known as Particle Swarm Optimization or Swarm Optimization, is the most popular Swarm Optimization algorithm in recent years. Kennedy and R. C. Evolutionary Algorithm based on Evolutionary Algorithm Eberhart and others. PSO is a kind of evolutionary algorithm, similar to simulated annealing algorithm, it also starts from the random solution and finds the optimal solution through iteration, it also evaluates the quality of the solution through fitness, but it is simpler than the rules of genetic algorithm, it does not have Crossover and Mutation operations of genetic algorithm, and it searches for the global optimum by following the optimal value of the current search. The Algorithm is easy to implement, high precision, fast convergence and so on, which has attracted the attention of academia and has shown its advantages in solving practical problems. Particle swarm optimization is a parallel algorithm.

Particle Swarm Optimizer, or Particle swarm optimization 1, is a swarm intelligence based optimization algorithm inspired by artificial life and social psychology. It was proposed by American social psychologists Kennedy and electrical engineer in 1995, the basic concept is derived from the study of birds and fish predation. PSO Algorithm is widely used in power systems, machine learning, pattern recognition, image processing and so on because of its advantages of a simple concept, easy implementation and free of the restriction of the problem-solution space. The motion equation of the first particle in the D dimensional space of the PSO algorithm can be described by a set of difference equations, as shown in Formulas 12 and 13:

$$
V_{i d}(\mathrm{t}+1)=\omega V i d(\mathrm{t})+C 1 R 1(\mathrm{t})\left(P_{i d}(\mathrm{t})-X_{i d}(\mathrm{t})\right)+C 2 R 2(\mathrm{t})\left(P_{g d}(\mathrm{t})-X_{i d}(\mathrm{t})\right)
$$


Jin Li and Fang Xie

$$
X_{i d}(\mathrm{t}+1)=X_{i d}(\mathrm{t})+V_{i d}(\mathrm{t}+1)
$$

Among them: $\operatorname{Xid}(\mathrm{t})$ and $\operatorname{Vid}(\mathrm{t})$ represent the position and velocity component of the

D dimension of the first particle; $P_{i d}(\mathrm{t})$ is the pbest historical position experienced by the

Individual Particle; $P_{g d}(\mathrm{t})$ is the gbest component of the best historical position experienced by the population; $C 1$ and $C 2$ are the acceleration factors, and $R 1$ and $R 2$ are random numbers distributed evenly between 0 and 1 , which are called random factors. The velocity update equation of the Algorithm can be divided into three items: the first item is momentum, which indicates the Inertia Movement of the particle at the previous velocity; the second item is cognition, which indicates the thinking of the particle itself; and the third item is society, represents information sharing and cooperation between particles.

\section{Principal Component Feature Extraction}

Principal component feature extraction is carried out on rearranged biological self-organization criticality and thermodynamic feature data information flow of a life system by adopting a neural network training method. Assuming that the neural network trained by the biological self-organization criticality and thermodynamic feature data sample set of the life system has a total of neurons, the neurons in the input layer and the neuron set in the hidden layer of the neural network are adaptively weighted according to the degree of weight degeneration, and their weight values are recorded as $\left\{W_{L}\right\}_{i=1}^{N-m}$. Under appropriate inertia weight value training, the state of the time biological self-organization criticality and thermodynamic feature data information flow of the life system can be recorded as follows:

$$
x_{k}=\sum_{i=1}^{N} \overrightarrow{x_{k}^{i}} \overline{w_{k}}
$$

Copy the neuron set with high weight, normalize the weight at last, and count the state of biological self-organization criticality and thermodynamic characteristic data time series of life system at time and time can be recorded as follows:

$$
\hat{x}_{k}=\sum_{i=1}^{m} \frac{n_{i}}{N} x_{k}^{i}, \quad \sum_{i=1}^{m} n_{i}=N
$$

Statistical biological self-organization criticality and residual vector of thermodynamic characteristic data of life system, KL distance between phase trajectory of residual vector and neurons in input layer are:

$$
K L(x)=\sum_{i=1}^{m} \frac{n_{i}}{N} \ln \frac{n_{i}}{N w_{d}^{i}}
$$

Wherein $n_{i}$ is the statistical adoption number of all the weight values in the information flow of biological self-organization criticality and thermodynamic characteristics of the life system. Using a neural network optimization algorithm to resample the biological self-organized criticality after information rearrangement and the thermodynamic characteristics of the life system, the statistical characteristics are recorded as $\left\{W_{\text {final }}\right\}=\left\{\left\{W_{H}\right\},\left\{W_{C}\right\},\left\{W_{O}\right\}\right\}$. At this time, the state estimation of the biological self-organized criticality and thermodynamic characteristics of the life system prediction self-organized supervised learning is as follows: 
Jin Li and Fang Xie

$$
\begin{aligned}
& x_{F}^{i}=\frac{1}{N}\left\{\sum_{i=1}^{m} x_{H}^{i}+\sum_{i=1}^{N-m-a} x_{O}^{i}+\sum_{i=1}^{a} x_{C}^{i}\right\}=\frac{1}{N} \sum_{i=1}^{m+a} x_{H}^{i}+\frac{1}{N} \sum_{i=1}^{N-m-a} x_{O}^{i} \\
& =\frac{1}{N} \sum_{i=1}^{m+a} x_{H}^{i}+\frac{1}{N} \sum_{i=1}^{N-m-a} x_{S}^{i}\left(1-K d_{i}^{\max }\right)+\frac{1}{N} \sum_{i=1}^{N-m-a} K d_{i}^{\max } x_{L}^{i}
\end{aligned}
$$

Principal component feature extraction of biological self-organization criticality and thermodynamic features of a life system is carried out by adopting a forward three-layer PID neural network system, and the neuron weight $S$ of the output layer can be rewritten as follows:

$$
\begin{gathered}
\left\{W_{\text {final }}\right\}=\left\{x_{F}^{i}\right\}_{i=1}^{N}=\left\{\frac{1}{N} \sum_{i=1}^{m+a} ; x_{H}^{i}, \frac{1}{N} \sum_{i=1}^{N-m-a}\left(1-K d_{i}^{\max }\right) ;\right. \\
\left.x_{S}^{i}, \frac{1}{N} \sum_{i=1}^{N-m-a} K d_{i}^{\max } ; x_{L}^{i}\right\}
\end{gathered}
$$

Set

$$
f_{1}(x)=\frac{1}{N} \sum_{i=1}^{m+a} x_{H}^{i}+\frac{1}{N} \sum_{i=1}^{N-m-a} x_{S}^{i}\left(1-K d_{i}^{\max }\right)+\frac{1}{N} \sum_{i=1}^{N-m-a} K d_{i}^{\max } x_{L}^{i} f_{2}(x)=\sum_{i=1}^{N} x_{D}^{i} w_{D}^{i}
$$

Then under the intelligent optimization algorithm of neural network training, the statistical information of biological self-organization criticality and thermodynamic characteristics of life system principal component feature extraction is expressed as follows:

$$
\begin{aligned}
& K L^{\prime}=\sum_{i=1}^{m+a} \frac{1}{N} \ln \frac{1}{N w_{d}^{i}(H)}+\sum_{i=1}^{N-m-a} \frac{1}{N} \ln \frac{1-K d_{i}^{\max }}{N} \ln \frac{1-K d_{i}^{\max }}{N w_{d}^{i}(H)} \\
& +\sum_{i=1}^{N-m-a} \frac{1}{N} \ln \frac{K d_{i}^{\max }}{N} \ln \frac{K d_{i}^{\max }}{N w_{d}^{i}(L)}
\end{aligned}
$$

Ignoring the influence of disturbance information of high-weight neurons, the biological self-organization criticality of the output layer of the neural network and the extraction result of the thermodynamic characteristic principal component output characteristic of the life system is obtained as follows:

$$
\begin{gathered}
K L=\sum_{i=1}^{m+a} \frac{1}{N} \ln \frac{1}{N w_{d}^{i}(H)}+\sum_{i=1}^{N-m-a} \frac{1}{N} \ln \frac{1}{N} \ln \frac{1}{N w_{d}^{i}(H)}+0 \\
\sum_{i=1}^{m} n_{i}=\sum_{i=1}^{N}=N
\end{gathered}
$$

\section{Self-organized criticality of organisms optimized by particle swarm optimization and prediction of thermodynamic characteristics of life systems}

The extracted principal component features are subjected to feature screening and self-organization supervised learning by using an optimized particle swarm algorithm. The fluorescein value of the global optimal position searched by the particle swarm represents a feature information point for the prediction of biological self-organization criticality and thermodynamic features of a life system. The update rules of the search process are as follows:

$$
l_{i}(k)=(1-\rho) l_{i}(k-1)+\gamma f\left(x_{i}(k)\right)
$$

Where: $\rho$ is the volatilization coefficient of fluorescein distributed between $(0,1)$, $\gamma$ represents the global optimal fitness value. When the number of iterations reaches the preset maximum, the particle swarm performs optimization on the predicted convergence degree of the biological self-organization criticality and the thermodynamic characteristics of the life system in the moving process, and is calculated by the following formula:

$$
P_{i j}(k)=\frac{\left(l_{j}(k)-l_{i}(k)\right) \eta_{i j}(k)}{\sum_{j \in N_{i}(k)}\left(l_{j}(k)-l_{i}(k)\right) \eta_{i j}(k)}
$$


Wherein, $j \in N_{i}(k), N_{i}(k)=\left\{\left\|x_{j}(k)-x_{i}(k)\right\|<r_{d}(k)\right\}, \eta_{i j}(k)$ is a visibility factor for feature screening, which mainly reflects the spatial distribution distance from particle swarm to particle swarm $i$.

In order to predict and control the weight errors in the values of biological self-organization criticality and thermodynamic characteristics of life systems, particle swarm optimization algorithm is adopted for self-organization supervised learning, and the learning iteration formula is as follows:

$$
x_{i}(k+1)=x_{i}(k)+s\left(\frac{x_{j}(k)-x_{i}(k)}{\left\|x_{j}(k)-x_{i}(k)\right\|}\right)
$$

Where: $\|\vec{x}\|$ represents the norm of $\vec{x}$. Since the fitness $\mathrm{f}$ (xi) of the particle swarm I is better than the fitness $\mathrm{f}(\mathrm{pg}$ ) of the global extreme value $\mathrm{pg}$, the perception range of the particle swarm is adjusted, $r_{d}^{i}(k)$ represents the dynamic decision range of the SDF-only particle swarm at $0<r_{d}^{i}(k)<r_{s}$ the time to predict the biological self-organization criticality and the thermodynamic characteristics of the life system, and the characteristic separation iteration formula for predicting the biological self-organization criticality and the thermodynamic characteristics of the life system is obtained as follows:

$$
r_{d}^{i}(k+1)=\min \left\{r_{S}, \max \left\{0, r_{d}^{i}(k)+\beta\left(n_{i}-\left|N_{i}(k)\right|\right)\right\}\right\}
$$

Among them, $\beta$ represents the biological self-organization criticality and the neighborhood change rate of the global search of the life system, $n_{i}$ represents the fitness value of the biological self-organization criticality and the particle swarm of the life system, inertia weight is adopted to control the transfer quantity of the low-weight particle swarm, neural network training is carried out, and the weight of the output optimization training is obtained to meet:

$$
w_{D}^{i}>\frac{K d_{i j}^{\max }}{N}
$$

Where: $N$ is the total number of particle swarm. $d_{i j}^{\max }$ is the maximum Euclidean distance between particles. The characteristic information rearrangement is adopted to modify the prediction training degree of the biological self-organization criticality and the thermodynamic characteristics of the life system, and the obtained prediction result is output as follows:

$$
x_{O}^{i}=x_{S}^{i}+K d_{i}^{\max }\left(x_{L}^{i}-x_{S}^{i}\right)
$$

Wherein, $K=1 /\left\|x_{L}^{i}-x_{S}^{i}\right\|$. According to the above description, the pseudo-code to realize the prediction of biological self-organization criticality and thermodynamic characteristics of the life system is described as follows:

$$
\begin{aligned}
& \text { Host }=\left\{h_{1}, h_{2}, h_{3} \cdots h_{n}\right\} \\
& H t_{i}=\left\{t_{1}, t_{2} \cdots t_{n}\right\}
\end{aligned}
$$

if (nodetask is not NULL) // Judging whether the learning factors are empty or not 
Jin Li and Fang Xie

$$
\begin{aligned}
& \text { if }(m(i)==0) \\
& m(i)=1 ; \\
& \text { else } \\
& \text { for } i=1 \text { to } H t_{i} \text { do } \\
& \quad t_{i} \in(0,1) \\
& \quad \text { for } j=1 \text { to } m(i) d o \\
& \quad S\left(h_{i}, H_{j}\right)=\operatorname{Cos}\left(\pi S\left(h_{i}, H_{j}\right)\right) \\
& \quad x=j ; \\
& \text { endfor } \\
& \text { endfor } \\
& \text { C }\left(h_{i}\right)=H_{x} \\
& \text { endif }
\end{aligned}
$$

\section{Bioenergy, the law of thermodynamics of matter and information}

Life constantly absorbs material energy and information from the environment and constantly removes material and information with less energy to the environment, the waste of information thus causes the body to acquire negative values. It, together with bioenergy and substances, causes the self-organization of organisms in the body, including biological tissues and organs, to form automatically and to grow well. Therefore, the comprehensive changes and coordination of energy materials and information throughout all life processes are life activities, it is worthwhile to study the law of this mutual transformation.

Generally speaking, for the Open non-equilibrium state of the entropy of the life system can always be expressed:

$$
\Delta S=\Delta_{i} S+\Delta_{e} S
$$

In the formula, $\Delta_{e} S$ here means that the negative pick value thrown to the environment by living organisms $\Delta_{i} S$ is an entropy change caused by the random and irreversible process of biological molecules in living organisms. It is generally regular $\Delta S$ can be positive and negative, the specific value is determined by the internal and external conditions and different growth periods of the living things. If we take advantage of the extensiveness of the soil moisture, considering the non-equilibrium characteristics of the living things, the biological changes of the common living things are expressed as:

$$
\frac{d S}{d t}=\frac{d_{e} S}{d_{e} t}+\frac{d_{i} S}{d_{e} t}
$$

The change in the entropy of the organism above causes the change in the amount of information that the organism absorbs, which is directly related to the negative entropy, as defined by Shammon et Al.

$$
H=-K \Delta S
$$

Where $K$ is the scale factor. This formula shows that organisms get negative entropy values from the environment as well as information from the environment. If the whole body has a 
Jin Li and Fang Xie

negative value of biological entropy, the more information, Biological self-organization is more orderly, the vitality of life is stronger. The total amount of information possessed by a living thing should be equal to the $H_{e}$ obtained from the environment, the body exists including the Genetic Information $H_{i}$ and with the waste discharge of Information $H_{e}^{\prime}$ sum, $H=H_{e}+H_{i}+H_{e}{ }^{\prime}$. Since an organism is a non-equilibrium dynamical system, and the amount of information it contains varies from time to time, the amount of information it contains should be:

$$
\Delta H=\Delta_{i} H+\Delta_{e} H
$$

The change over time is:

$$
\frac{d H}{d t}=\frac{d_{e} H}{d_{e} t}+\frac{d_{i} H}{d_{e} t}
$$

This means that at a certain time the total amount of information that a living thing has changed with time is always equal to the amount of information per unit time from the outside world $\frac{d_{e} H}{d_{e} t}$ and the total amount of information that still exists in the body $\frac{d_{i} H}{d_{e} t}$, the amount and direction of information in Vivo and in vitro restrict the development and change of self-organization and indicate the degree and direction of the organization. For example, the metabolic process of life can be regarded as the change process of biological entropy and biological information. Life always absorbs energy high protein, fat and starch and other high-energy low-entropy high-order food, out of low-energy, high-entropy disorder large dross. Therefore, the process of metabolism of the living body always absorbs the low entropy material, excretes the high entropy waste, and obtains the negative entropy of the living body. The greater the negative entropy, the stronger the metabolism, the greater the vitality of life. The faster this process goes, the greater the change in entropy, the greater the negative entropy, and the more biological energy, matter, and information a living organism gets. It means that the more vital you are, the stronger your body is. And vice versa.

In addition, the changes in biological entropy and information described in (29) - (32) can be used to account for changes in the disease status of an organism. The quantitative calculation of biological entropy in the development of myocardial infarction is listed in Table 1. The biological entropy values of the mild and severe patients were listed in five stages.

Table1. Myocardial infarction patients are different, the same stage of the bio-digest value.

\begin{tabular}{ccccccc}
\hline $\begin{array}{c}\text { Biological } \\
\text { entropy }\end{array}$ & $\mathbf{1 \sim 2}$ & $\mathbf{3 ~ 7}$ & $\mathbf{8 \sim 1 5}$ & $\mathbf{1 6 ~ 3 0}$ & $\mathbf{3 1} \mathbf{6 0}$ & $\begin{array}{c}\text { Average } \\
\text { value }\end{array}$ \\
\hline $\begin{array}{c}\text { Light } \\
\text { patient } \\
\text { Severe }\end{array}$ & 1.902 & 2.032 & 2.105 & 2.005 & 1.916 & 1.992 \\
\hline
\end{tabular}




\section{patients}

It can be seen from the table that the biological entropy of the light patients at any time is always less than the entropy of the serious patients, but they are all greater than the biological entropy of the serum protein spectrum of the same age, the same sex and the same nationality healthy people at the same time. This indicates that the degree of order of the self-organized system, such as serum protein, is gradually destroyed, and the vitality and function of the organism are weakened. Therefore, we can use biological changes to reflect the severity of the disease. In addition, it can be seen from the above table that during the $60 \mathrm{~d}$ course of the disease, the bio-entropy value of the serum protein spectrum increased and decreased, and there was a peak value in the middle. The ascending phase showed that the degree of order of serum protein decreased greatly, and the descending phase showed that the degree of order was restored. The whole thing is curving. In the first two days, the biological entropy value rose greatly, which indicated that the disordering tendency strengthened, the condition develops quickly. After $3 \sim(8 \mathrm{~d})$ or $8 \sim(15 \mathrm{~d})$, the biological entropy increased greatly, which indicated that the disease got worse and then returned to some extent. These results show that it is possible to scientifically calculate the change of biological entropy in order to diagnose the development of a patient's disease. Now to study the energy and material in the body of time changes in the law. If the energy and mass of the life system are $E_{1}$ and $M_{1}$, and the energy and material of the waste discharged by the

living body to the environment are $E_{2}$ and $M_{2}$, then there are

$$
d H=E_{1}+E_{2}, d M=M_{1}+M_{2}, d H=H_{1}-H_{2}
$$

According to the characteristics of life, the open system is called:

$$
d E+\lambda d M=d u_{0}+P d V+d u(d E, d M)
$$

Among them, $u_{0}$ is internal energy, $P$ and $V$ are pressure and volume, and $d u$ is the energy that the living body itself dissipates to maintain biological self-organization. Obviously, it's $d E, d M, P$ and $V$ are functions, and the upper formula can be rewritten as:

$$
\frac{d u}{d t}=\left(\frac{d E}{d t}+\lambda \frac{d M}{d t}\right)_{e}-\left(\frac{d u_{0}}{d t}+P \frac{d V}{d t}\right)_{i}=\frac{d_{e} u}{d t}-\frac{d_{i} u}{d t}
$$

$\frac{d_{e} u}{d t}=\left(\frac{d E}{d t}+\lambda \frac{d M}{d t}\right)_{e}$ represents the energy value of, per unit time obtained from the outside in the process of energy exchange between the system and the outside environment. $\frac{d_{i} u}{d t}=-\left(\frac{d u_{0}}{d t}+P \frac{d V}{d t}\right)_{i}$ indicates the irreversible process of the transformation from an ordered state to a disordered state, and the amount of dissipative energy per unit time (36). 
The formula indicates that the energy dissipated by an organism in an external environment with a biological self-organization structure in order to maintain the self-organization of the organism so that it can survive is not only restricted and controlled by the internal factors of the system, are also subject to the impact and constraints of the external environment. If $\frac{d_{e} u}{d t}>\frac{d_{i} u}{d t}$ and $\frac{d u}{d t}>0$ at this time, the living body has enough energy material to maintain and improve the biological self-organization, so that the living body continues to grow and develop when $\frac{d_{e} u}{d t}=\frac{d_{i} u}{d t}$ and $\frac{d u}{d t}=0$, ordered self-organization has ceased to develop, life is in a critical state and a third state. When $\frac{d_{e} u}{d t}<\frac{d_{i} u}{d t}$ and $\frac{d u}{d t}<0$, shows that the self-organization of living things began to dissipate the energy stored by themselves, and then to decline, collapse direction. When you're $\frac{d_{e} u}{d t}=0$, you tend to die. Thus, the dialectical relationship between biomass energy and matter expressed by formula (36) does reflect the basic conditions of the growth, development and change of living organisms.

Thus (30), (32) (33) and (36) together can comprehensively reflect the energy, material, biological entropy and information changes in the life system and the basic characteristics. It is different from the traditional formula of the first and second laws of thermodynamics. It is a special and new law of complex life systems. For this, we call it a new law of thermodynamics. This law not only expresses the law of the whole change of energy, matter, biology and information in the life system, at the same time, it also reflects the law that the system's state and character change with time, that is, the dynamic change of the system and the changing relationship between them, that is, but it can also express the relationship between the growth, development, change and decrepitude of life. Thus, it is a fundamental law of life. In principle, it can be used to discuss the law and characteristics of all changes and development of the life system or life body, as well as the biological and physiological phenomena it produces. Also, this law applies, in principle, to any open Non-equilibrium thermodynamics system that is characterized by dissipative structure or self-organization.

\section{Analysis of Simulation Test Results}

The performance test of the biological self-organization criticality and thermodynamic characteristic prediction model of the life system is carried out through simulation experiments. The population size of the particle swarm is set to be $m=40$, dimension $D=30$, inertia weight $\omega=0.98$, the search range of neural network training is $--5.24 \leq x_{d} \leq 5.24$, the training function is selected to be that the number of neuron nodes of the neural network is $h=4,8$, the total number of iterations is 10,000 , the fluorescein sensing coefficient of the particle swarm, According to the above simulation environment and parameter setting, six groups of biological self-organization criticality and thermodynamic characteristic data samples of the life system are taken to predict the biological self-organization criticality and thermodynamic characteristic of the life system, and the sample mean square error (RMSE) comparison is performed to define:

$$
\operatorname{RMSE}\left(\bar{x}_{k}\right)=\sqrt{\frac{1}{N_{M C}} \sum_{i=1}^{N_{M C}}\left(x_{k}^{i}-\bar{x}_{k}\right)^{2}}
$$

The evolution map of the self-organized criticality of organisms and thermodynamic characteristics of life systems thus obtained is shown in figure 1. 
Jin Li and Fang Xie

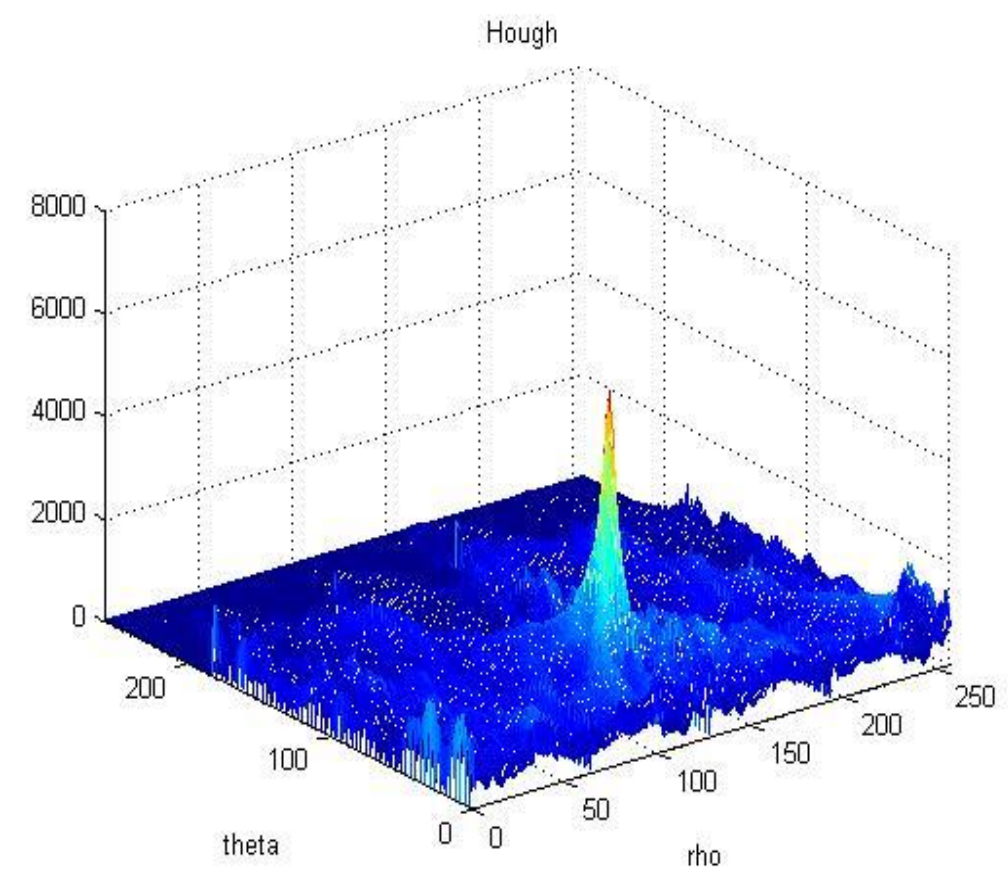

Figure 1. Evolution map of biological self-organization criticality and thermodynamic characteristics of the life system.

The model is used to predict the thermodynamic characteristics of biological self-organization criticality and life system, and the prediction error comparison results are shown in Figure 2.

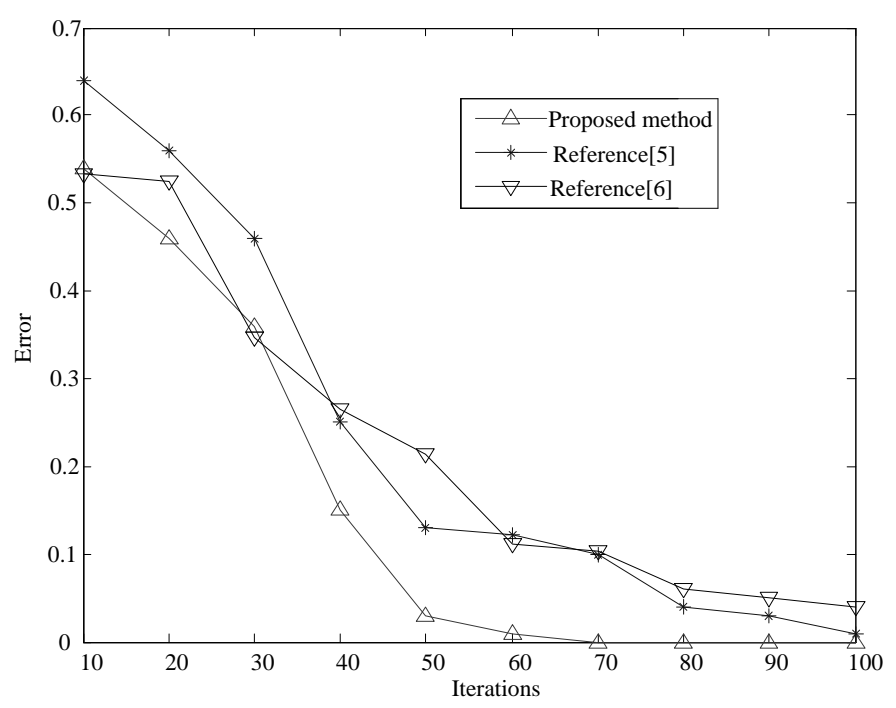

Figure 2. Comparison results of prediction errors of thermodynamic characteristics between biological self-organization criticality and life system.

Analysis of the results in fig. 2 shows that the mean square error for predicting the self-organized criticality of organisms and thermodynamic characteristics of life systems using the model in this paper is relatively low. Table 1 shows the time and accuracy improvement results of Cell Mol Biol (Noisy le Grand) 2020 | Volume 66 | Issue 2190 
Jin Li and Fang Xie

different algorithms for the prediction of biological self-organization criticality and thermodynamic characteristics of life systems. The improved accuracy is based on the particle swarm prediction model as the reference standard. It is known that the running time of this model is the shortest, $0.2913 \mathrm{~s}$, and accuracy improvement is the highest at $63.15 \%$. The prior sample knowledge of biological self-organization criticality and thermodynamic characteristics of life systems is relatively small, only 300 sets of sample data are needed. The experimental results show the superiority of this model.

Table 2. Performance Comparison of Different Algorithms.

\begin{tabular}{cccc}
\hline Algorithm type & Number of Prior Samples & Run time & Accuracy improvement \\
\hline $\begin{array}{c}\text { PSO } \\
\begin{array}{c}\text { Ant colony } \\
\text { algorithm }\end{array}\end{array}$ & 600 & 0.3456 & - \\
$\begin{array}{c}\text { Fuzzy neural } \\
\text { network }\end{array}$ & 800 & 0.3435 & $14.43 \%$ \\
Colony & 1000 & 0.3567 & $22.56 \%$ \\
This method & 1200 & 0.7434 & $23.35 \%$ \\
\hline
\end{tabular}

\section{Conclusions}

In this paper, a prediction model of biological self-organization criticality and thermodynamic characteristics of the life system based on particle swarm optimization neural network is proposed. Fuzzy regression parameter fusion model is adopted to rearrange the statistical prior data of biological self-organized criticality and thermodynamic characteristics of life system, neural network training method is adopted to extract principal component characteristics of rearranged biological self-organized criticality and thermodynamic information flow of life system, and optimized particle swarm algorithm is adopted to carry out feature selection and self-organized supervised learning on extracted principal component characteristics, thus realizing accurate prediction of biological self-organized criticality and thermodynamic characteristics of life system. The simulation results show that the prediction accuracy of biological self-organization criticality and thermodynamic characteristics of the life system using this model is high, and the prior sample knowledge required is relatively small, with reliability and accuracy.

\section{References}

1. LEE G M, LEE J H. On nonsmooth optimality theorems for robust multiobjective optimization problems. J Nonlinear Convex Anal 2015; 16(10): 2039-2052.

2. Sun X K, Peng Z Y, Guo XL. Some characterizations of robust optimal solutions for uncertain convex optimization problems. Optim Lett 2016; 10(7): 1463-1478.

3. Lee GM, Lee JH. On nonsmooth optimality theorems for robust multiobjective optimization problems, J Nonlinear Convex Anal 2015; 16(10): 2039-2052.

4. Sun X K, Peng Z Y, Guo XL. Some characterizations of robust optimal solutions for uncertain convex optimization problems, Optim Lett 2016; 10(7): 1463-1478. 
5. Fakhar M, Mahyarinia M R, Zafarani J. On nonsmooth robust multiobjective optimization under generalized convexity with applications to portfolio optimization, European J Opera Res 2018; 265(1): 39-48.

6. Sun X K, Li X B, Long X J, et al. On robust approximate optimal solutions for uncertain convex optimization and applications to multi-objective optimization, Pacific J Opt 2017; 13(4): 621-643.

7. Han J, Yuan J, Wei X, Lu Y. Pedestrian visual positioning algorithm for underground roadway based on deep learning, J Comput Appl 2019; 39(3): 688-694.

8. Yu Z, LI L, LIU J, et al. Adaptive noise immune cluster ensemble using affinity propagation, IEEE Transact Knowledge Data Eng, 22015: 7(12): 3176-3189.

9. Huang D, Lai J, Wang C. Combining multiple clusterings via crowd agreement estimation and multi-granularity link analysis, Neurocomput, 2015; 17: 240-250.

10. Yang Y. Elements of information theory, J Am Statist Asso 2008; 103(3): 429-429.

11. Iam-On N, Boongoen T, Garrett S, et al. A link-based approach to the cluster ensemble problem, IEEE Transactions on Pattern Analysis and Machine Intelligence, 2011; 33(12): 2396-2409.

12. Xu J, Miao D, Zhang Y, et al. A three-way decisions model with probabilistic rough sets for stream computing, International Journal of Approximate Reasoning, 2017; 88; 1-22.

13. SU Kuanhao, HU Lingzhi, STEHNING C, et al. Generation of brain pseudo-CTs using an undersampled,single-acquisition UTE-mDixon pulse sequence and unsupervised clustering, Med Physic 2015; 42(8): 4974-4986.

14. Johansson A, Karlsson M, Nyholm T.Ct substitute derived from MRI sequences with ultrashort echo time, Medical Physics 2011; 38(5): 2708-2714.

15. Johansson A, Karlsson M, Yu Jun, et al. Voxel-wise uncertainty in CT substitute derived from MRI, Medical Physics, 2012; 39(6): 3283-3290. 